\title{
ARTICLE
}

\section{Investigation of development condition with new nuclear emulsion for neutron measurement under high gamma-ray background}

\author{
Kohei Ishihara ${ }^{\mathrm{a}}$, Jun Kawarabayashi ${ }^{\mathrm{a}}$, Hideki Tomita ${ }^{\mathrm{a}}$, Tatsuhiro Naka ${ }^{\mathrm{b}}$, Takashi Asada ${ }^{\mathrm{b}}$, \\ Kunihiro Morishima ${ }^{\mathrm{b}}$, Mitsuhiro Nakamura ${ }^{\mathrm{b}}$, Shigetaka Maeda ${ }^{\mathrm{c}}$ and Tetsuo Iguchi ${ }^{\mathrm{a}}$ \\ ${ }^{a}$ Graduate School of Eng., Nagoya Univ., Furo-cho, Chikusa-ku, Nagoya, 464-8603, Japan ; ${ }^{b}$ Graduate School of Sci., Nagoya Univ., \\ Furo-cho, Chikusa-ku, Nagoya, 464-8603, Japan; ' Japan Atomic Energy Agency, Oharai-cho, Higashiibaraki-gun, Ibaraki, \\ 311-1393, Japan
}

In order to measure neutron flux and neutron energy distribution under high intensity gamma-ray background, we tried to reduce the efficiency for a gamma-ray with our original nuclear emulsion by falling in development temperature whereas keeping efficiency for a neutron. The emulsion was irradiated by neutron and gamma-ray, and developed with Developer for Opera Experiment at temperatures of 10, 15 and $20{ }^{\circ} \mathrm{C}$. As a result, the efficiency for gamma was successfully reduced with keeping the efficiency for a neutron, but tiny fog increased with increasing developing time using Developer for Opera Experiment.

\section{Keywords: nuclear emulsion; gamma background; neutron detector}

\section{Introduction}

The spent nuclear fuel needs to be shielded enough to decrease exposed dose not only by gamma-ray but also by neutron. In order to design a effective shielding, it is essential to investigate the neutron characteristics emitted from the spent fuel such as an emission rate, an emission profile and an energy spectrum with high precision. However, it is difficult to measure the characteristics of neutrons from the spent fuel assembly precisely because it emits much gamma-ray from containing fission products and its neutron/gamma ratio reaches to the order of $10^{-8} \sim 10^{-6}$. Currently, the neutron characteristics from the spent fuel are estimated by a simulation based on its burning history. But, the measurement data of neutrons is important in order to improve the reliability of the shielding calculation.

A nuclear emulsion [1], which was used in the earliest days in nuclear science, is a solid detector composed of silver halide and gelatin, which can record the tracks of charged particles. It is possible to discriminate neutrons from gamma-rays because the shapes of the track of the recoil proton which is formed by the neutron and the track of the electron which is formed by the gamma-ray are different each other (Figure 1). Also, it was used to measure neutron spectra at startup in the nuclear fuel [2]. So, we have proposed to apply the nuclear emulsion for the neutron measurement from the spent fuel. In this work, we reported the best development condition to reduce the efficiency for the gamma-ray to suppress background

*Corresponding author. Email: kawarabayashi@nagoya-u.jp

gamma signals.

(a)

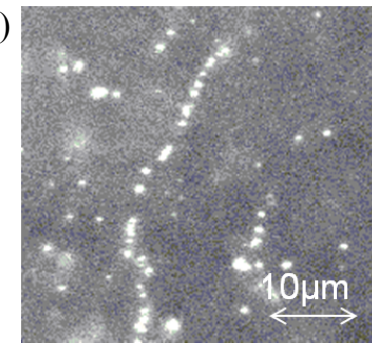

Figure 1. Microscopic image of non-sensitized OPERA film. There is the track perpendicular to the film which was formed by recoil proton (a) and electron (b).

\section{New nuclear emulsion}

As described previously, it is possible to discriminate neutrons from gamma-rays with the nuclear emulsion. But, it is difficult to recognize the neutron track in emulsion exposed to lots of gamma-rays because of a lot of electron tracks drown out the tracks of neutrons (Figure 2). So the efficiency for the gamma-ray needs to be reduced as low as possible whereas keeping the efficiency for the neutron.

We made a new nuclear emulsion, which we call it Neutron Gamma Image TrAcker emulsion (NGITA emulsion), in which the grain size of $\mathrm{AgBr}$ was controlled to be 60,90 and $160 \mathrm{~nm}$ in diameter based on non-sensitized OPERA emulsion to reduce the efficiency to gamma-ray, because the sensitivity of 
emulsion, which is a correlation between stopping power and silver grain density, depends on the $\mathrm{AgBr}$ size. The sensitivity estimated experimentally is reported in ref. [3]. Also, the neutron efficiency is reported in ref [3]. We used one type of these emulsions with grains of 60 $\mathrm{nm}$ in diameter without any sensitization.

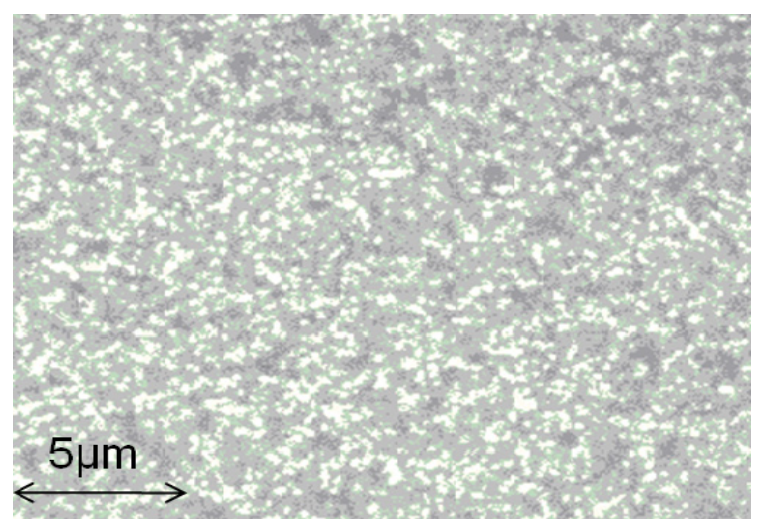

Figure 2. Microscopic image of the developed emulsion with gamma-ray fluence of $10^{12} \mathrm{\gamma} / \mathrm{cm}^{2}$.

\section{System of development}

A latent image speck composed of some silver atoms is produced on $\mathrm{AgBr}$ grains when a charged particle pass through in an emulsion. The size of latent image speck depends on the amount of deposited energy in a $\mathrm{AgBr}$ grain, and the developing speed depends on this size illustrated shown in Figure 3. Also, there is a threshold number of silver atoms in latent image specks to trigger the development of the $\mathrm{AgBr}$ grain. Development rate and the threshold number of silver atoms depend on developing condition (for example, developer temperature, $\mathrm{pH}$, etc) [4].

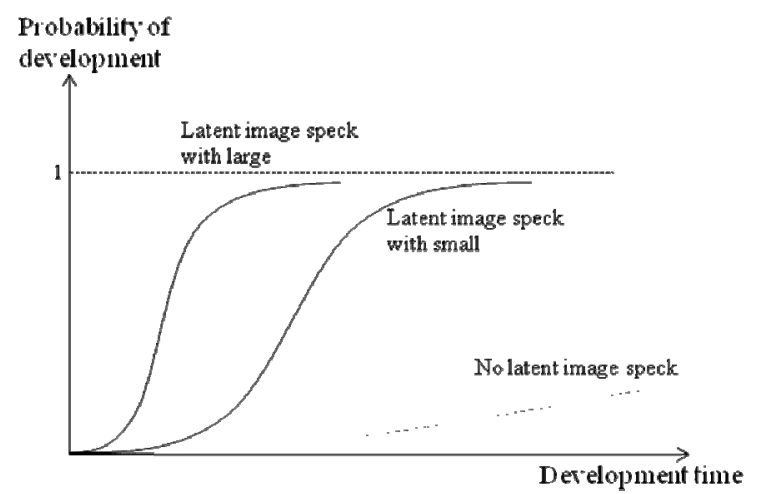

Figure 3. The relationship between the probability that $\mathrm{AgBr}$ with latent image speck is developed and development time [4].

The latent image speck produced by the proton is larger than that by the electron because the stopping power of a proton is larger than that of an electron. From this point of view, it is expected that only an $\mathrm{AgBr}$ grain irradiated with a proton can be developed by controlling the development condition.

\section{Experiment and result}

NGITA emulsion films with $\mathrm{AgBr}$ size of $60 \mathrm{~nm}$ was irradiated by neutron from ${ }^{252} \mathrm{Cf}$ source $\left(8.9 \times 10^{2} \mathrm{kBq}\right)$ for about one day at room temperature, and developed with Developer for Opera Experiment in temperatures of 10,15 and $20^{\circ} \mathrm{C}$. The emulsion films used were prepared by coating with NGITA emulsion on a base made of slide glasses. A neutron source was attached to the emulsion film shown in Figure 4. The number of grains $(\mathrm{N})$ consisting of the track, which was length of 8 $\mu \mathrm{m}$, in the developed emulsion film was measured by using a microscope. The silver grain density (G.D) was evaluated by the following equation.

$$
\mathrm{G} . \mathrm{D}=\mathrm{N} / 8
$$

The experimental results are shown in Figure 5. From these results, the development rate decreased with lowering the development temperature. In each temperature, the grain density was saturated with increasing developing time at which all latent image speck were developed. The saturated time is the best development time for each temperature to obtain the proton tracks.

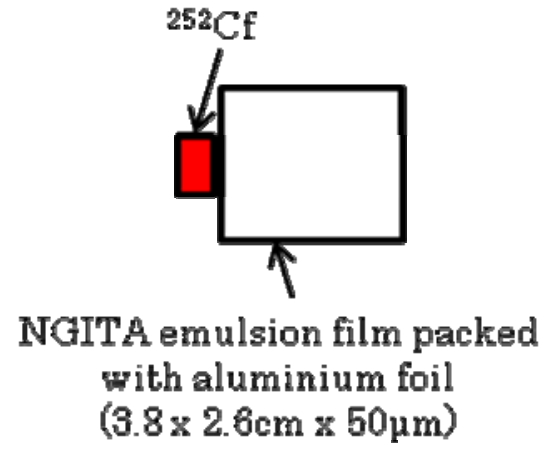

Figure 4. The experimental setup for neutron irradiation. The emulsion was set to be parallel to neutron incident direction.

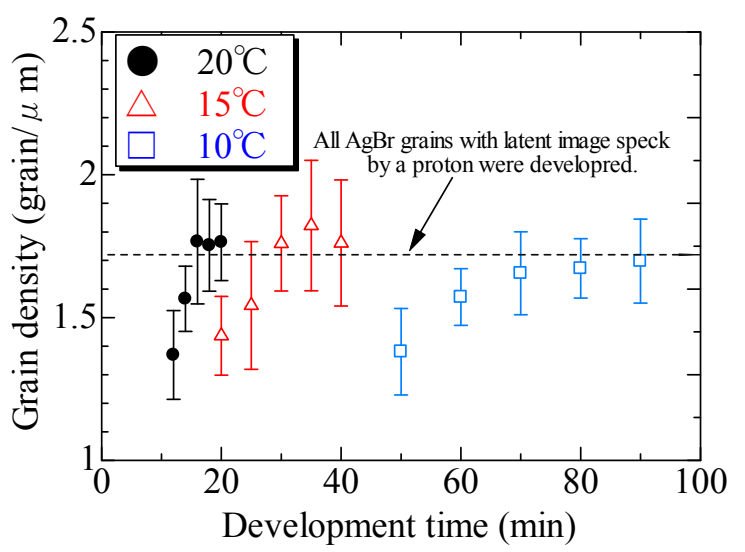

Figure 5. The grain density of new emulsion with $\mathrm{AgBr}$ size of $60 \mathrm{~nm}$. 
The NGITA emulsion films were irradiated by gamma-ray from ${ }^{60} \mathrm{Co}$ source $(54 \mathrm{TBq})$ at the ${ }^{60} \mathrm{Co}$ irradiation facility of Nagoya University, and developed in the same condition. The emulsion film is positioned $140 \mathrm{~cm}$ from the source (at center absorbed dose rate : $0.63 \mathrm{~Gy} / \mathrm{h}$ (water) ) such as in Figure 6 and irradiated for $10 \mathrm{sec}$ at room temperature. Also, another emulsion films with no gamma-ray irradiation was developed. The results of the developed irradiated emulsion films by gamma-ray are shown Figure 7 and non-irradiated one are shown in Figure 8. The number of grains formed by the incident gamma-ray which was obtained by subtracting the number of developed grains in the non-irradiated emulsion film is shown in Figure 9. These results indicate that the sensitivity to gamma-ray irradiation decreases with lowering the development temperature. However, the tiny fog, which is formed by development of $\mathrm{AgBr}$ grains without any latent image specks, increased with prolonging the development time as shown in Figure 8. As a result, total number of silver grains formed for the best development time at $10^{\circ} \mathrm{C}$ was larger than that of at $15^{\circ} \mathrm{C}$ and $20^{\circ} \mathrm{C}$ shown in Figure 7. The tiny fog can't be suppressed by decreasing the development temperature because the mechanism of development of tiny fog may be different from that of $\mathrm{AgBr}$ with latent image speck. In order to resolve this problem, it is necessary to find the developer that can suppress the tiny fog by decreasing the development temperature.

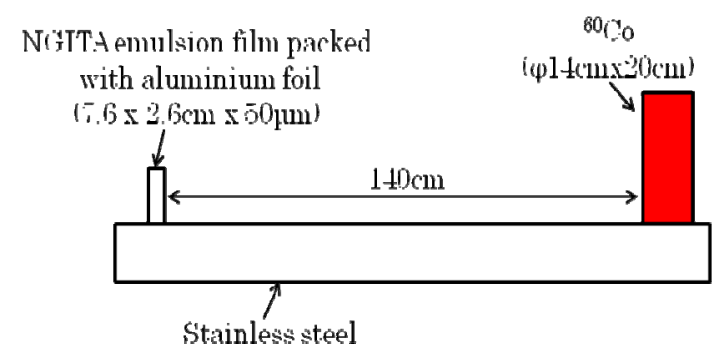

Figure 6. The experimental system for gamma-ray irradiation.

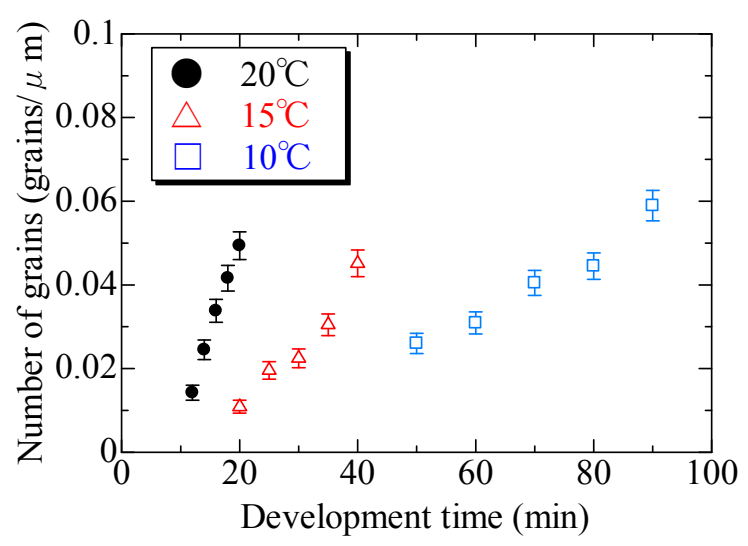

Figure 7. The number of grains in NGITA emulsion film with $\mathrm{AgBr}$ size of $60 \mathrm{~nm}$ when it was irradiated by gamma-ray fluence of $2.2 \times 10^{10} \mathrm{\gamma} / \mathrm{cm}^{2}$.

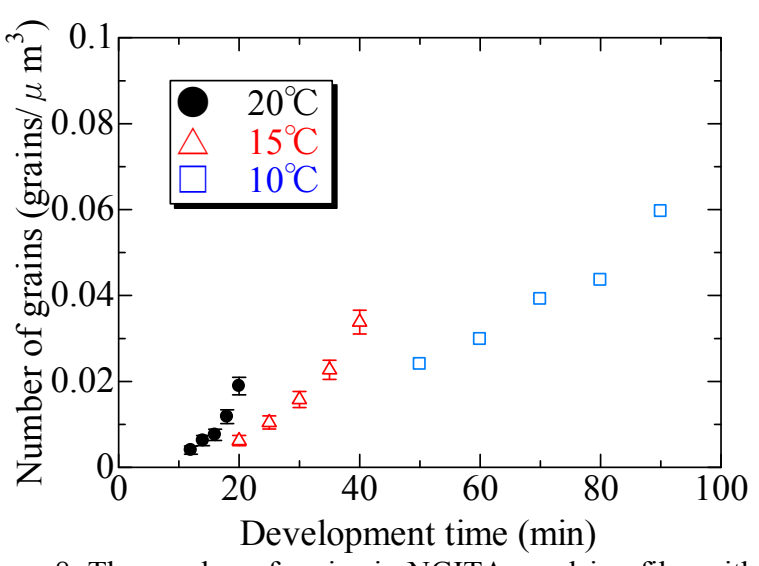

Figure 8. The number of grains in NGITA emulsion film with $\mathrm{AgBr}$ size of $60 \mathrm{~nm}$ when it was not irradiated.

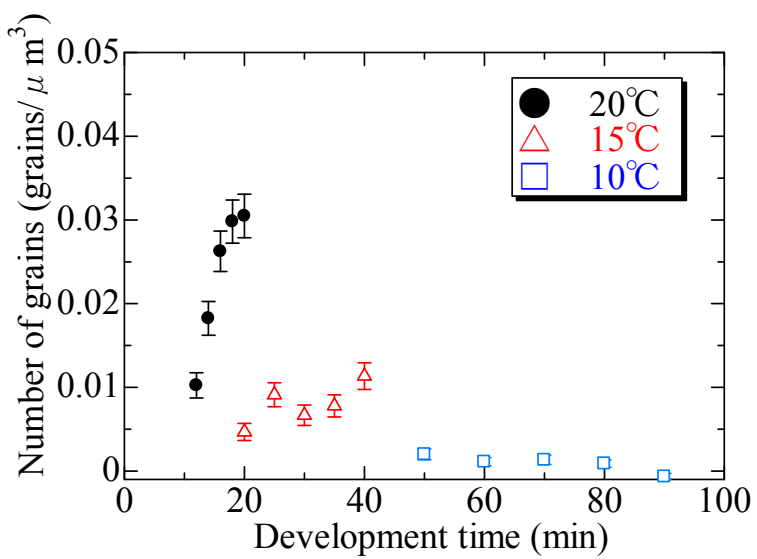

Figure 9. The number of grains for gamma-ray in NGITA emulsion film with $\mathrm{AgBr}$ size of $60 \mathrm{~nm}$ when it was irradiated by gamma-ray fluence of $2.2 \times 10^{10} \gamma / \mathrm{cm}^{2}$.

\section{Conclusion}

We made new nuclear emulsion (NGITA emulsion) based on non-sensitized OPERA film in order to detect the neutron under high gamma-ray background such as the spent fuel assembly. By lowering the developer temperature, the efficiency for the gamma-ray could be reduced whereas keeping the efficiency for the neutron. But, this method of development with Developer for Opera Experiment increased the tiny fog with increasing development time.

As a future work, we will investigate most suitable developer with this method of development that generates few tiny fog.

\section{References}

[1] N. Armenisea, M. De Serio, M. Ieva et al., High-speed particle tracking in nuclear emulsion by last-generation automatic microscopes, Nucl. Inst. Meth. Phy A, 551, 2-3(2005), pp.261-270.

[2] R. Gold and J.M. Roberts, Nuclear emulsion neutron spectrometry in the FFTF, Trans. Am. Nucl. 
Soc., 39(1981), pp.892-904.

[3] K. Ishihara, K. Takagi, H. Minato, J. Kawarabayashi, H. Tomita, S. Maeda, T. Naka, K. Morishima, T. Nakano, M. Nakamura and T. Iguchi, Fast neutron detection under intense gamma-ray fields with novel nuclear emulsion technique, Radiation Measurements (to be published).

[4] K. Kuge and H. Kobayashi, Chemistry of Photograph, Maruzen, Japan, (2001), pp. 81-83. ISBN4621049135 